\title{
Control estructural para el emplazamiento de vetas y domos félsicos en el distrito minero de Zacatecas, México
}

\author{
Margarito Tristán-González ${ }^{1, *}$, José Ramón Torres Hernández, \\ Guillermo Labarthe-Hernández ${ }^{1}$, Alfredo Aguillón-Robles ${ }^{1}$; Roberto Yza-Guzmán ${ }^{2}$ \\ ${ }^{1}$ Instituto de Geología, Universidad Autónoma de San Luis Potosí, Av. Dr. Manuel Nava No. 5, Zona Universitaria, San Luis Potosí, \\ San Luis Potosí, México 78240. \\ ${ }^{2}$ Compañía Minera Capstone Gold S.A. de C.V., Priv. Loma Baja No. 101, fraccionamiento Loma, Zacatecas, Zacatecas, México \\ 98068. \\ *mtristan@uaslp.mx
}

\begin{abstract}
Resumen
El distrito minero de Zacatecas (DMZ) fue considerado como uno de los yacimientos de plata más importantes desde el tiempo de la Colonia hasta fines del siglo XIX. Actualmente las operaciones mineras son atractivas, debido al hallazgo de nuevos sitios con mineralización económica rentable. La zona de estudio está localizada al norte de la ciudad de Zacatecas, donde afloran secuencias marinas del Triásico, sedimentos vulcano-sedimentarios del Cretácico Temprano, rocas clásticas y volcánicas del Eoceno-Oligoceno. La secuencia del Mesozoico fue afectada, al menos, por dos etapas de deformación compresiva que originó plegamiento y fallamiento inverso, ocasionando imbricación de gran parte de la secuencia. Estos eventos de deformación tuvieron lugar principalmente durante el Cretácico y principio del Cenozoico y son más conspicuos en los sedimentos vulcano-sedimentarios. Después del último evento compresivo correspondiente a la orogenia Laramide, ocurrió una fase de deformación transtensiva que seccionó la sierra en cinco bloques, separados por las fallas La Cantera, Mala Noche, Veta Grande, Pánuco y Casa de Cerros. En este trabajo se propone un modelo para el emplazamiento de las vetas y domos félsicos del DMZ, basado en un control estructural de movimiento transtensivo y extensivo. El sistema de vetas y fallas desarrolladas en la etapa transtensiva presentan un arreglo escalonado con desarrollo de fracturas Riedel y de "cola de caballo" en los extremos de las fallas. La cinemática de esta etapa de fallamiento está documentada con los indicadores cinemáticos que están impresos en los planos de las mismas, donde se observan dos juegos superpuestos de estrías: uno horizontal (derecho) y otro oblicuo. Esto sugiere que el segundo evento de fallamiento normal aprovechó los planos de ruptura generados en el primer evento (lateral). Las estrías horizontales impresas en los planos de falla de la roca de la UVS y sobre las cuales se sobrepusieron las vetas de cuarzo, muestran que las fallas y fracturas generadas en la primera etapa transtensiva fueron los conductos para el ascenso del magma que dio origen a los diques y domos félsicos, y a la primera etapa de mineralización en las vetas principales. Las fallas y fracturas generadas en la primera etapa transtensiva, fueron los conductos para el ascenso del magma que dio origen a los diques y domos félsicos de la riolita Bufa. El segundo evento de reactivación de las fallas fue normal oblicuo izquierdo, lo cual es evidente en la falla La Cantera. El domo La Bufa está emplazado en una zona de dilatación de esta falla durante su primera etapa de actividad y posteriormente afectado por el evento de extensión con asociación de eventos hidrotermales que causó alteraciones (argilización y silicificación) en la zona de falla. Los cuerpos minerales más importantes se localizan en los escalones de las fallas donde se formaron las zonas dilatación de las cuatro fallas principales formadas por la transtensión.

La zona estudiada se encuentra dentro de la región de la provincia de Cuencas y Sierras (CyS), y se caracteriza por la presencia de fosas, semifosas y pilares tectónicos desarrollados principalmente durante el Oligoceno. Las fallas que seccionaron esta porción norte de la sierra de Zacatecas tienen un rumbo promedio $\mathrm{N} 60^{\circ} \mathrm{W}$, las cuales forman parte del sistema de fallas propuesto como San Luis-Tepehuanes.
\end{abstract}

Palabras clave: Sierra de Zacatecas, vetas, fallas laterales, transtensión. 


\begin{abstract}
The Zacatecas mining district was considered one of the most important silver deposits from colonial times until the end of the XIX century; mining operations are currently attractive, as they have found new sites with profitable economic mineralization. The study area is located north of the city of Zacatecas, where marine Triassic sequences, volcano-sedimentary deposits of the Early Cretaceous, clastic and volcanic rocks of the Eocene crop out. The Mesozoic rocks were affected by at least two stages of compressive deformation associated with folding and reverse faulting, which caused imbrication of most of the sequence. These deformation events occurred mainly during the Cretaceous and early Tertiary and are more conspicuous in the volcano-sedimentary deposits. After the last compressive event of the Laramide orogeny, a transtensive deformation phase dissected the mountain into five blocks separated by La Cantera, Mala Noche; Veta Grande, Pánuco and Casa de Cerros faults. In this paper we propose a model for the emplacement of the veins and felsic domes of the Zacatecas mining district based on structural control by transtensional and extensional movement. The fault and vein systems have an echelon arrangement with development of reidel fractures and horsetail patterns at fault terminations. Movement along the faults is supported by kinematic indicators on the fault planes, where there are two overlapping sets of grooves: one horizontal (dextral) and one oblique. This suggests that a second event of normal faulting took place along the rupture planes generated during the first event (lateral). First stage faults and fractures provided conduits for mineralization in the principal veins and the ascent of felsic dikes and domes. The second fault reactivation event was normal-left oblique, which is evident in La Cantera fault and corresponds to the second stage of mineralization. The Bufa dome was emplaced in the dilation zone of this fault during the first stage of activity, and was later affected by hydrothermal events during extension, causing alteration (argilization and silicification) in the fault zone. The ore bodies are found mainly in dilation zones of the four main fault steps formed by transtension.

The study area is located within Basin and Range Province, and is characterized by the presence of basins, half grabens, and horsts developed mainly during the Oligocene. Faults that dissected the Zacatecas range have an average orientation of N6 ${ }^{\circ} \mathrm{W}$, coinciding with the system known as San Luis-Tepehuanes.
\end{abstract}

Keywords: Sierra de Zacatecas, veins, strike-slip faults, transtension.

\section{Introducción}

El norte de la sierra de Zacatecas (SZ) forma parte de la región límite entre las provincias fisiográficas de la Sierra Madre Occidental (SMO) y la Mesa Central (MC). En su porción norte se ubica el distrito minero de Zacatecas, considerado en el siglo pasado como uno de los centros mineros más importantes en la producción de plata y oro, así como de plomo, zinc y cobre. Los yacimientos se alojan en vetas hidrotermales, sobresaliendo las vetas $\mathrm{La}$ Cantera, Mala Noche, Veta Grande y Pánuco-Casa de Cerros (Figura 1). Su bonanza minera se inició en el tiempo de la Colonia y concluyó a fines del siglo XIX. La geología de estos yacimientos se ha descrito por varios autores entre los que sobresalen Stone (1956); Bastin (1941); Mapes (1949); Pérez-Martínez et al. (1961); Ranson (1975); Ocejo-Pérez (1979) y Ponce-Sibaja (1985). Un trabajo de síntesis fue publicado recientemente por el Servicio Geológico Mexicano (1999) (Caballero-Martínez et al., 1999). Actualmente la actividad minera está renaciendo y recientes trabajos de exploración directa han derivado en la ubicación de nuevos cuerpos mineralizados alojados en vetas, sobresaliendo la de Mala Noche explotada por la Compañía Minera Capstone Gold S. A. de C. V., cuyo concentrado principal es de cobre.

La geología del Mesozoico de la SZ es compleja, en especial en los alrededores de la ciudad de Zacatecas donde se ha reconocido una secuencia estratigráfica que incluye rocas marinas metamorfoseadas del Triásico Tardío y una secuencia vulcano-sedimentaria asociada a un arco volcánico de edad Jurásico Tardío-Cretácico Temprano (Burckhardt y Scalia, 1906; Gutiérrez-Amador, 1908; Pérez-Martínez et al., 1961; McGhee, 1976) que descansa discordantemente sobre la secuencia metamórfica triásica (Yta, 1992; Tristán-González et al., 1994; CentenoGarcía y Silva-Romo, 1997; Escalona-Alcázar et al., 2009). La secuencia vulcano-sedimentaria, además cubre tectónicamente a los sedimentos triásicos. La secuencia del Cenozoico se inició con los lechos rojos del Eoceno que fueron reconocidos por Edwards (1955) en gran parte del centro de México, aflorando en toda la porción centro y oriente de las ciudades de Zacatecas y Guadalupe, donde consiste de una secuencia de conglomerado polimíctico y estratos de limo y arcilla depositados discordantemente sobre la unidad vulcano-sedimentaria y rellenando una fosa tectónica (Tristán-González, 2008). La secuencia volcánica cenozoica dentro del DMZ inició con la erupción de oleadas piroclásticas y flujos de ceniza. Esta actividad volcánica explosiva precedió a la emisión de flujos extensos de lava que formaron domos a través de diques emplazados a lo largo de los sistemas de fallas y fracturas.

La porción de la SZ que se encuentra al norte de la ciudad de Zacatecas está seccionada por cuatro fallas de orientación NW-SE: La Cantera, Mala Noche, Veta Grande y Pánuco, que se han reportado por autores diversos (Bastin, 1941; Pérez-Martínez et al., 1961; Ponce-Sibaja y Clark, 1988). 


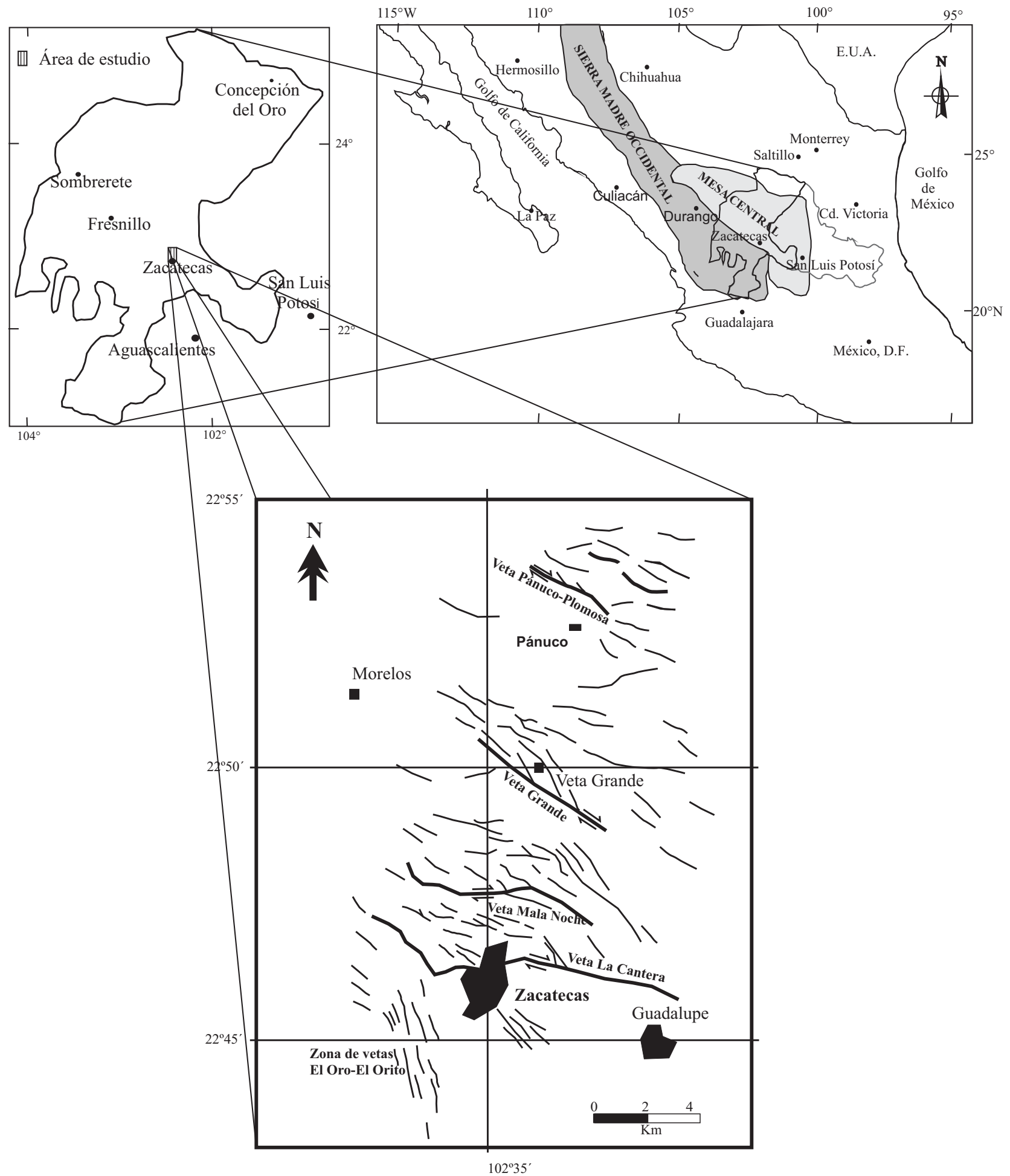

Figura 1. Localización del área, en la porción norte de la ciudad de Zacatecas. En el inserto inferior se muestra el arreglo de los cuatro sistemas de vetas del Distrito Minero de Zacatecas, tomado de Ponce-Sibaja y Clark (1988).

Los indicadores cinemáticos en los planos de estas fallas y el patrón de fracturas asociadas (fracturas Riedel), se generaron por movimiento lateral derecho. Los principales domos y diques félsicos (riolita Bufa) se emplazaron en estas estructuras. Posteriormente, estas fallas se reactivaron por efecto de esfuerzos extensionales de orientación NE$\mathrm{SW}$, generando fallas normales escalonadas de dirección NW-SE, cortando y basculando a la lava de la riolita Bufa (Tristán-González, 2008).

Se han hecho varios intentos para explicar el origen 
de los yacimientos minerales del DMZ, relacionándolos preferentemente a fallas normales y/o domos riolíticos, o a una caldera. En la primera hipótesis, los domos riolíticos ( $\sin$ tomar en cuenta las ignimbritas) y el sistema de fallas-vetas del distrito minero se han relacionado con una tectónica extensional donde las fallas normales permitieron el emplazamiento de los domos, y aportaron la permeabilidad para el desarrollo de sistemas hidrotermales durante el proceso de emplazamiento del magma riolítico que quedo atrapado a profundidad, formando las vetas mineralizadas en el distrito. En la segunda hipótesis, el sistema de fallas, distribución de domos y diques e ignimbritas se acomodan en un modelo semicircular no muy bien definido, proponiéndose una caldera, la de Zacatecas (Ponce-Sibaja y Clark, 1988).

El presente trabajo aporta el análisis estructural de la porción norte de la SZ entre la ciudad de Zacatecas y el poblado Pánuco-Casa de Cerros, cubriendo un área de aproximadamente $150 \mathrm{~km}^{2}$ (Figura 1). De este análisis se ha podido documentar que las fallas del DMZ y vetas asociadas, son el resultado de dos eventos de deformación: el primero de naturaleza transpresiva y que controló además el emplazamiento de los domos félsicos, y el segundo de naturaleza extensional, que basculó los bloques previamente delimitados por fallas laterales en el primer evento de deformación. El objetivo de este trabajo es contribuir al conocimiento de la deformación post-laramídica que afectó a la porción norte de la SZ y analizar su relación con los domos félsicos y yacimientos minerales asociados que se encuentran en el DMZ.

\section{Geología}

\subsection{Estratigrafía local}

\subsubsection{Formación Zacatecas.}

Las rocas marinas en las inmediaciones de la ciudad de Zacatecas fueron reportadas por Burckhardt (1906), donde reportó restos fósiles que fueron la base para establecer la edad del Triásico Tardío. Posteriormente Burckhardt y Scalia (1906), describieron a detalle la geología a lo largo del arroyo La Calavera (arroyo La Pimienta o El Bote), donde separaron las pizarras sericíticas de la base y las silicificadas del nivel superior, donde separaron las pizarras sericíticas de la base y las silicosas del nivel superior, mencionando que las pizarras del nivel inferior pertenecen al Triásico tardío, con base en los fragmentos de cefalópodos (Sirenites Smith; Trachyceras; Clionites sp. y Juvavites (Anatomites) mojsvari) y bivalvos encontrados en la parte superior de este paquete.

Posteriormente McGhee (1976) subdividió esta secuencia en cinco unidades, de la más antigua a la más joven: A) filita en su mayor parte, con variaciones a esquisto y pizarra. Esta unidad contiene pelecípodos y amonites; B) capas gruesas de cuarcita gris y de filita que alternan con capas de meta-arenisca, donde se han reportado trazas de circón, apatita y minerales opacos; C) capas de filita de color gris y laminaciones de pedernal. Hacia arriba se intercalan filitas de color gris y verde, con esquisto negro. La cima es una secuencia de capas de filita de color morado claro, meta-pedernal, meta-arenisca de feldespato color gris; D) alternancia de capas delgadas de meta-arenisca de color gris y filita gris, con apariencia de flysch y, localmente, metaconglomerado, meta-arenisca masiva y filita/pizarra; E) secuencia en donde predominan las capas de filita y esquisto de color gris, y en menor cantidad esquisto blanco y negro.

Monod y Calvet (1992) hicieron una reinterpretación estratigráfica de esta secuencia y dividieron a la Formación Zacatecas en tres unidades: Formación Pimienta, constituida por capas de caliza, aglomerado de carácter vulcaniclástico, material tobáceo y esquisto sericítico, que culminan en su cima con capas de conglomerado y toba blanca correspondientes a las unidades C y E de McGhee (1976); Formación El Bote, es una alternancia de capas de metalutitas negras y capas de arenisca cuarcífera, que corresponde a la unidad D de McGhee op.cit. y Formación El Ahogado, constituida por una alternancia de capas delgadas de metalutita de color negro y arenisca cuarcífera, equivalente a las unidades A y B de McGhee op.cit.

La edad de la Formación Zacatecas (Triásico Tardío) fue determinada desde los primeros estudios de Burckhardt (1906). Posteriormente, autores como Gutiérrez-Amador (1908), encontraron nuevas variedades de fósiles de crinoides, moluscos, fragmentos de huesos grandes de reptiles del Mesozoico y ejemplares de Palaeoneilo sp; Pleurotoma sp. y Juvavites sp., que sitúan a estos sedimentos como de edad Cárnico. Maldonado-Koerdell (1948) identificó en los afloramientos de pizarras que afloran en el arroyo La Pimienta, fósiles como Rhynchonella sp. y un ejemplar de Nucula sp., pertenecientes al Triásico Tardío.

Centeno-García y Silva-Romo (1997) interpretaron que la secuencia de la Formación Zacatecas se depositó en un talud continental, y la consideraron como parte de la porción oriental del Terreno Guerrero. Consideraron además que por su composición geoquímica, corresponden a depósitos de un ambiente de piso oceánico, y que estos marcan la margen continental occidental de México para el Triásico Tardío-Jurásico Medio.

\subsubsection{Unidad Vulcano-Sedimentaria (UVS)}

Esta unidad está compuesta de una serie de "rocas verdes" que sobreyacen discordante y tectónicamente a la secuencia metamórfica de edad triásica (Burckhardt y Scalia, 1906; Gutiérrez-Amador, 1908; Pérez-Martínez et al., 1961; McGhee, 1976). El nombre de Unidad VulcanoSedimentaria (UVS) fue propuesto por Yta (1992). La composición más común de la UVS es una secuencia de lavas andesíticas muchas de las cuales llegan a presentar estructura almohadillada y están intercaladas con sedimentos terrígenos donde predominan las capas de arenisca masiva y filita. Toda la secuencia de lavas y sedimentos terrígenos 
está cortada por diques, dique-estratos y apófisis pequeños de roca gabroica. Centeno-García y Silva-Romo (1997) nombraron a esta secuencia como formación La Borda y la describen como una sucesión de flujos de lava basáltica intercalados con capas de arenisca de grano fino de origen volcánico, toba, pedernal verde y lutita negra. Estos autores mencionan que las formaciones La Borda y Zacatecas están cabalgadas.

Uno de los estudios más recientes sobre esta unidad fue realizado por Escalona-Alcázar et al. (2009); ellos nombraron a esta secuencia como Complejo Vulcanosedimentario Las Pilas, y la describieron como una sucesión de lavas máficas, tobas escasas, diques, sills y lacolitos, intercalados con horizontes de grauvaca feldespática, mudstone y lentes de caliza. Las lavas con estructura de almohadillas comúnmente están foliadas y/o deformadas. La mineralogía de las lavas consiste de fenocristales de plagioclasa, clinopiroxeno y poco olivino; la matriz varía de microcristalina a criptocristalina. La lava tiene alteración hidrotermal y deutérica intensa, lo que promovió la formación de sericita, calcita, epidota y clorita. La mineralogía de los intrusivos (diques, sills y lacolitos) es muy parecida a la de las lavas, prácticamente con los mismos minerales. Los minerales máficos están alterados a clorita y la plagioclasa a calcita y sericita, la textura es fanerítica, porfirítica y poikilítica. El espesor de los diques puede alcanzar $10 \mathrm{~m}$ y pueden cambiar a sills, los cuales pueden tener $2 \mathrm{~m}$ de espesor y extenderse hasta $1.5 \mathrm{~km}$. Las calizas ocurren en lentes, y generalmente están intercaladas con las lavas en almohadilla deformadas.

La UVS se puede correlacionar con la Formación Chilitos que aflora en el arroyo del mismo nombre, en la inmediación oriente de la mina de Fresnillo, Zacatecas (de Cserna, 1976), y con los afloramientos del arroyo El Gallinero en el poblado El Saucito, Zacatecas, muy cerca del límite con el estado de San Luis Potosí (Yta, 1992).

$\mathrm{Su}$ edad se obtuvo por medio de la fauna encontrada en el arroyo Chilitos, en las inmediaciones de la mina de Fresnillo, en donde se colectaron fósiles en lentes de caliza, intercalados entre lavas andesíticas almohadilladas. El rango de edad de estos fósiles abarca desde el Titoniano hasta el Hauteriviano (de Cserna, 1976). Yta (1992) reportó radiolarios en la caliza pelágica intercalada entre el basalto almohadillado en los afloramientos de la UVS de El Saucito, Zacatecas, lo que permitió situar su edad en el Cretácico Temprano. Escalona-Alcázar et al. (2009) también obtuvieron una edad del Cretácico Temprano con circones detríticos e incluyeron en esta edad a la secuencia que le subyace, la cual se ha considerado desde los estudios de Burckhardt (1906) como del Triásico Tardío. La edad reportada por Escalona-Alcázar et al. (2009) es contradictoria con la fauna reportada en el arroyo $\mathrm{La}$ Pimienta por Burckhardt (1906) y Gutiérrez-Amador (1908), por lo que es posible que se hayan muestreado circones en algunos de los múltiples ensambles de la UVS que se encuentran sobre las rocas metamórficas de la Formación Zacatecas.

\subsubsection{Conglomerado Rojo de Zacatecas}

En discordancia sobre la UVS, aflora en los alrededores de la ciudad de Zacatecas una secuencia de lechos rojos. Este depósito fue estudiado por Botsford (1909) quien lo nombró como "conglomerado Guanajuato". Posteriormente Stone (1956) lo denominó "aglomerado Zacatecas", por su semejanza con el depósito que se encuentra en el distrito minero de Guanajuato estudiado por Edwards (1955). En este trabajo se utiliza el nombre de conglomerado rojo de Zacatecas, utilizado por Ponce-Sibaja y Clark (1988).

En la ciudad de Zacatecas este conglomerado tiene en los primeros $25 \mathrm{~m}$, guijarros con diámetro variable entre 2 y $10 \mathrm{~cm}$. De mayor a menor abundancia, los clastos están compuestos de rocas volcánicas silicificadas (roca verde), brechas volcánicas, cuarzo lechoso, calcedonia, granito, caliza y arenisca; algunas capas están bien cementadas, pero la mayor parte del depósito basal está incluido en una matriz arcillo-arenosa de color rojizo o verdoso.

Hacia la parte media y superior hay capas con poca o nula compactación, donde el tamaño de las guijas pueden alcanzar los $30 \mathrm{~cm}$, con redondez mediana a bien desarrollada, donde predominan los fragmentos de andesita y arenisca de la secuencia vulcano-sedimentaria, cuarzo lechoso de veta, granito, caliza y pedernal. En los afloramientos donde se observa la parte superior, es un depósito de fragmentos finos $(2-5 \mathrm{~cm})$ en una matriz de arena o limo.

El depósito en general presenta horizontes lenticulares con gradación normal y estratificación cruzada, con abundantes paleocauces, lo que representa una alta tasa de depósito. La matriz tiene por lo general tonos rojizos por el óxido de hierro.

$\mathrm{Su}$ espesor es variable dentro de la fosa de Zacatecas, disminuye hacia el noroeste y desaparece en el Centro Histórico de Zacatecas. En los sitios donde aflora con el mayor grosor, Edwards (1955) calculó un espesor de 133 m y Stone (1956) lo estimó en $200 \mathrm{~m}$.

Estos depósitos descansan en forma discordante sobre la UVS y subyacen a los volcaniclásticos Los Alamitos, riolita Bufa e ignimbrita La Virgen. La edad del conglomerado rojo de Zacatecas fue estimada por palinología por NájeraGarza (1997), como del Paleoceno-Eoceno temprano. El conglomerado rojo de Zacatecas se correlaciona con depósitos semejantes que afloran en otras localidades de Guanajuato y San Luis Potosí, donde varios autores han fechado lavas intercaladas entre ellos, p. ej. Aranda-Gómez y McDowell (1998) para Guanajuato y Labarthe-Hernández et al. (1982); Tristán-González y Torres-Hernández (2000), Tristán-González et al. (2009) y Torres-Hernández et al. (2000), para localidades en el estado de San Luis Potosí.

\subsubsection{Volcaniclásticos Los Alamitos.}

Ponce-Sibaja y Clark (1988) nombraron a los flujos piroclásticos que se encuentran debajo de la riolita Bufa, como "volcaniclásticos Los Alamitos". Esta unidad es la más antigua del área; en su porción basal consiste de oleadas 
piroclásticas que hacia arriba pasan a un flujo parcialmente soldado rico en líticos (30\%); estos líticos tienen diámetro hasta de $10 \mathrm{~cm}$ de rocas volcánicas, además de fragmentos de pómez amarillenta parcialmente colapsada. En la cima del flujo disminuye el contenido de líticos y está parcialmente soldada. Ponce-Sibaja y Clark (1988) determinaron una edad isotópica por K-Ar en biotita, de 46.8 Ma.

\subsubsection{Riolita Bufa}

Pérez-Martínez et al. (1961), agruparon a todas las rocas félsicas del $\mathrm{DMZ}$ como riolitas y pórfidos riolíticos. Tristán-González (2008) agrupó a la serie de domos y diques félsicos como riolita Bufa.

La riolita es de color gris claro, contiene escasos fenocristales de sanidino (1-2\%), de 1 a $2 \mathrm{~mm}$ de largo, con menor proporción de cuarzo, escasa biotita y hematita diseminada. La matriz está devitrificada y tiene una trama fluidal. Presenta gran número de juntas verticales con depósito fumarólico de óxidos de hierro. La mayor parte de la roca está muy silicificada, argilizada y brechada. Otros domos localizados en la porción norponiente del domo La Bufa (La Sierpe, El Magistral, San Gil; Figura 3) se encuentran muy silicificados. Se obtuvo una edad por el método K-Ar de roca completa de $40.5 \pm 0.9 \mathrm{Ma}$ (Tabla 1).

\subsubsection{Ignimbrita La Virgen}

Sobre las lavas de la riolita Bufa y el conglomerado Zacatecas, se depositó una secuencia de flujos piroclásticos que afloran al sur de la ciudad de Zacatecas, el cual fue nombrado por Pérez-Martínez et al. (1961) como "riolitas". Posteriormente, Ponce-Sibaja y Clark (1988) denominaron informalmente a estos depósitos como formación La Virgen. En este trabajo se dividió esta unidad en dos secuencias claramente distinguibles en el campo. La secuencia inferior consiste de depósitos de oleadas piroclásticas con líticos pequeños, la cual va pasando gradualmente a un flujo de ceniza de color café y pómez sin compactar, rica en fragmentos líticos $(40 \%)$, culminando en la cima con una brecha lítica. La secuencia superior de esta ignimbrita inició como un flujo de ceniza parcialmente soldado, de color gris rosáceo, con pómez bien compactada y fenocristales de cuarzo, sanidino y biotita (máximo 5\%). La parte superior de esta secuencia es de alto grado de soldadura e incluye evidencias de reomorfismo. La roca es de color pardo grisáceo, con 5\% de fenocristales de cuarzo, sanidino, biotita, pómez muy compactada y líticos aislados. Su matriz está devitrificada y presenta estructura fluidal. Ponce-Sibaja y Clark (1988) reportaron una edad por el método K-Ar en sanidino de 36.8 Ma, para una muestra tomada en la Mesa La Virgen en la inmediación sur de la ciudad de Zacatecas. Para este trabajo se obtuvo una edad por el método K-Ar en roca entera de $37.1 \pm 0.9 \mathrm{Ma}$ (Tabla 1) para una muestra de la Mesa La Virgen, cerca de la antena de microondas (ZA-2, coordenadas geográficas $22.745^{\circ}-101.429^{\circ}$, Tabla 1). Esta unidad fue considerada por Ponce-Sibaja y Clark (1988) como parte de la ignimbrita intra-caldera de la "caldera de Zacatecas".

Para la identificación de la secuencia estratigráfica del área se puede consultar la Figura 2.

\section{Marco Tectónico}

La porción norte de la SZ se ha interpretado como un horst limitado por fallas normales con orientación N-NE y N-NW (Caballero-Martínez et al., 1999; Loza-Aguirre et al., 2008; Tristán-González, 2008). La parte más elevada de la SZ alcanza los 2700 m s.n.m. en el sur de la ciudad de Zacatecas (cerro La Virgen). El valle al oriente de la sierra, tiene una altitud de 2100 m s.n.m. donde afloran rocas del Terreno Guerrero y remanentes de rocas félsicas y básicas (domos, ignimbritas y basaltos). El valle al poniente de la SZ tiene una altitud promedio de 2200 m s.n.m., donde afloran sedimentos del Terreno Guerrero y remanentes de rocas félsicas, principalmente ignimbritas.

Campa-Uranga y Coney (1983) sitúan a la SZ en la porción oriental del Terreno Guerrero, el cual está compuesto por rocas volcánicas, volcaniclásticas y calizas que comprenden la secuencia conocida como Formación Zacatecas y UVS. La Formación Zacatecas representa es una secuencia depositada sobre un piso oceánico parcialmente metamorfizado, mientras que la UVS consiste de rocas derivadas de un arco de islas (Centeno-García y SilvaRomo, 1997).

De acuerdo con el análisis estructural de la secuencia vulcano-sedimentaria, la tectónica de acortamiento ocurrida durante el Mesozoico en esta porción del Terreno Guerrero es más compleja que la ocurrida en el Terreno Sierra Madre localizado al oriente (Centeno-García y Silva-Romo, 1997). La secuencia de la UVS fue afectada por fallas de

Tabla 1. Nuevas edades K-Ar para rocas volcánicas félsicas del distrito Minero de Zacatecas.

\begin{tabular}{|c|c|c|c|c|c|c|c|c|}
\hline \multirow{2}{*}{ Unidad Volcánica } & \multirow{2}{*}{ Muestra } & \multicolumn{2}{|c|}{ Coordenadas } & \multirow{2}{*}{$\begin{array}{l}\text { Edad } \\
\pm 1 \sigma^{\S}\end{array}$} & \multirow{2}{*}{$\begin{array}{c}{ }^{40} \mathrm{Ar}^{*} \\
(\%)\end{array}$} & \multirow{2}{*}{${ }^{40} \mathrm{Ar}_{\mathrm{R}}{ }^{\#}$} & \multirow{2}{*}{$\begin{array}{c}\mathrm{K}_{2} \mathrm{O} \\
\text { (wt. \%) }\end{array}$} & \multirow{2}{*}{ Fracción** } \\
\hline & & Latitud N & Longitud W & & & & & \\
\hline Ignimbrita La Virgen & $\mathrm{ZA}-2$ & $22.745^{\circ}$ & $101.429^{\circ}$ & $37.1 \pm 0.9$ & 77.1 & 92.25 & 7.64 & $\mathrm{RE}$ \\
\hline Riolita Bufa & ZB-1 & $22.775^{\circ}$ & $96.561^{\circ}$ & $40.5 \pm 0.9$ & 91.1 & 91.7 & 6.95 & RE \\
\hline
\end{tabular}

Edades realizadas en el Laboratorio de Geocronología de la Universidad de Bretaña Occidental, Brest, Francia.

$\S$ Error en un $\sigma$ fue calculado con la ecuación dada por Cox y Dalrymple (1967)

${ }^{40} \mathrm{Ar} *$; Contenido de Argón radiogénico de la muestra, en por ciento del total.

\# ${ }^{40} \mathrm{ArR}$; El Argón radiogénico en la muestra expresado en $10^{-7} \mathrm{~cm}^{3} / \mathrm{g}$.

** Material fechado; RE-roca entera 


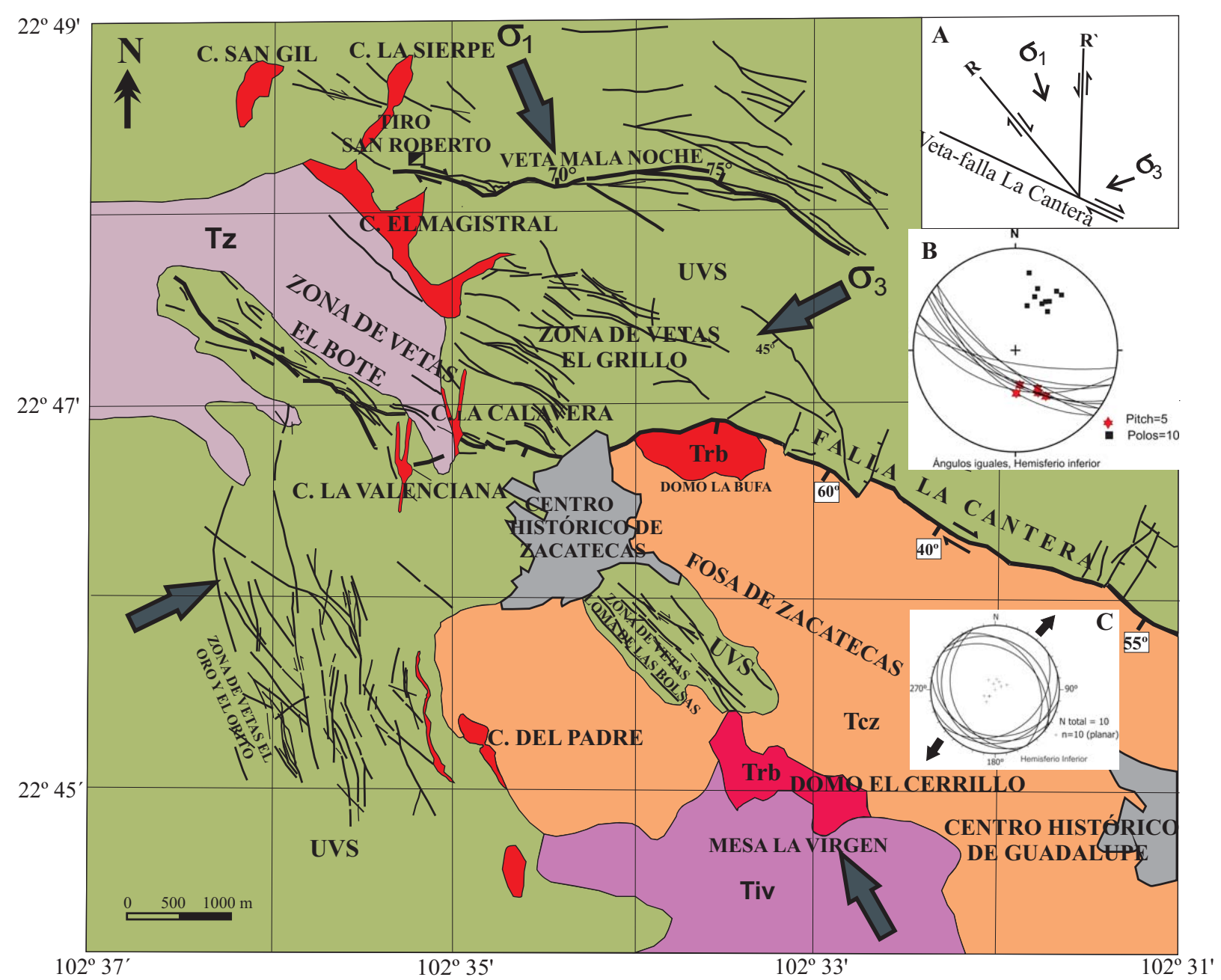

Figura 2. Mapa estructural del la zona de la veta-falla La Cantera y Mala Noche. El desarrollo de los patrones de fracturas, muestran un arreglo dextral generando fracturas $\mathrm{R}$ y R'. Los domos riolíticos del Eoceno medio en color rojo, están emplazados sobre el patrón de fracturas resultantes Riedel. En el inserto (A) es un esquema cinemático de la Veta La Cantera. En el estereograma inserto (B), se representan segmentos de la Veta La Canteara con su pitch al SE, y en el estereograma (C) el basculamiento del conglomerado rojo de Zacatecas. Tz- Formación Zacatecas; UVS-Unidad Vulcano Sedimentaria; Tcz- conglomerado rojo de Zacatecas; Tiv- Ignimbrita La Virgen (modificado de Pérez-Martínez et al., 1961).

despegue importantes hacia el oriente, a partir del Jurásico Medio y Tardío (Centeno-García y Silva-Romo, 1997). A esta tectónica se sobrepuso la deformación Laramide en el Mesozoico tardío, por lo que el acortamiento de la secuencia en esta área es muy significativo, colocando secuencias de la UVS sobre sí mismas. En la periferia poniente de la ciudad de Zacatecas, en el flanco occidental de la sierra, afloran las rocas triásicas de la Formación Zacatecas. Esta unidad muestra cuando menos tres períodos de deformación compresiva: una de dirección $315^{\circ}-320^{\circ}$, la otra en dirección $050^{\circ}-060^{\circ}$ (la cual está acorde al acortamiento laramídico) y una tercera representada por un conjunto de ejes orientados $360^{\circ}$ con evidencias de ser el resultado de una compresión con dirección al poniente (Tristán-González et al., 1994). Sobre el arroyo La Pimienta, al oriente del cerro El Gato, la UVS se encuentra cabalgando a la Formación Zacatecas (Tristán-González et al., 1994).

Escalona-Alcázar et al. (2009) analizaron el estilo de deformación para las rocas de la Formación Zacatecas y el Complejo Volcanosedimentario Las Pilas (UVS) a partir de un análisis geométrico de foliaciones, obteniendo orientaciones promedio de sus polos de $235^{\circ} / 86^{\circ}$ y $244^{\circ} / 86^{\circ}$ respectivamente. El análisis de paleoesfuerzos para las fallas inversas, resultó en un valor del vector $\sigma_{1}$, de $210^{\circ} / 01^{\circ}$, lo cual sugiere que la foliación y el fallamiento inverso fue contemporáneo durante la orogenia Laramide. Estas actitudes de los polos y paleoesfuerzos son semejantes a los obtenidos por Tristán-González et al. (1994) para los afloramientos del arroyo La Pimienta. Escalona-Alcázar et al. (2009) en su análisis de fallas normales, encontraron una orientación promedio de $029^{\circ} / 00^{\circ}$ para el vector $\sigma_{3} \mathrm{y}$ señalaron que las fallas normales cortan a las inversas e interpretaron que esta fallas se desarrollaron en un evento post-laramídico.

La SZ se ubica en la porción sur-poniente de la provincia de Cuencas y Sierras (CyS) de México en el límite con 
la provincia fisiográfica de la Sierra Madre Occidental (SMOc) (Stewart, 1978, 1998; Henry y Aranda-Gómez, 1992). La provincia de CyS se caracteriza por la presencia de sistemas de fallas normales desarrolladas al menos en cuatro fases de extensión a partir del Oligoceno temprano. Estas fases están ligadas a eventos contemporáneos de vulcanismo que ocurrieron después de un lapso breve de fallamiento (Aranda-Gómez et al., 2000), los cuales están asociadas a pulsos de actividad tectónica-magmática que coinciden en el tiempo con el incremento en la tasa de divergencia entre las placas Farallón y Pacífica, o a períodos de reorganización de las mismas en la costa del Pacífico (Aranda-Gómez et al., 2000). Por otro lado, Nieto-Samaniego et al. (1999) sugirieron, de acuerdo a las relaciones estratigráficas de las unidades volcánicas de la región de San Luis Potosí y Guanajuato, que la extensión pudo haber ocurrido a partir del Eoceno, identificando tres fases de extensión: la primera ocurrió antes de $32 \mathrm{Ma}$, la segunda entre 30 y $27 \mathrm{Ma}$ y la tercera entre 24 y $11 \mathrm{Ma}$, las cuales estuvieron acompañados de actividad volcánica intensa. La zona de Zacatecas está afectada por fallas normales, propuestas por Nieto-Samaniego et al. (2005) como parte del sistema de fallas San Luis-Tepehuanes. Este sistema está localizado en el límite entre la SMOc y la provincia fisiográfica de la Mesa Central, identificado desde San Luis de La Paz, Guanajuato hasta Tepehuanes, Durango y lo dividieron en cinco tramos, cada uno de ellos con sus características propias. El tramo que comprende este estudio, corresponde a la sierra de Zacatecas, donde se han identificado dos sistemas de fallas (Ponce-Sibaja y Clark, 1988; Loza-Aguirre, 2005); las fallas más antiguas tienen un rumbo NW-SE con $50^{\circ}-70^{\circ} \mathrm{SW}$ y corresponden a las vetas mineralizadas, La Cantera, Mala Noche, Veta Grande, Plomosas y Pánuco (Ponce-Sibaja y Clark, 1988); las fallas NW-SE están cortadas por la fallas más jóvenes con rumbo N-S, que limitan la SZ y la configuran como un pilar tectónico. A estas fallas las consideraron como normales. En su trabajo también argumentan que la actividad de este sistema de fallas ha actuado desde antes del Oligoceno. Loza-Aguirre et al. (2008) consideraron que en la zona de la intersección del sistema de fallas San Luis-Tepehuanes y el graben de Aguascalientes, estuvo afectada por dos períodos de deformación: el primero ocurrió durante el Paleoceno-Eoceno temprano, generando fallas normales de rumbo WNW y NNE. Ellos mencionan que en este evento se emplazaron las vetas y se formó el graben de Zacatecas donde se depositó el conglomerado rojo. El segundo periodo ocurrió durante el Oligoceno tardío y Mioceno temprano, generando fallas de rumbo NNE y WNW que al reactivarse desplazaron las vetas del DMZ.

\section{Sistema de fallas de la sierra de Zacatecas}

La última fase de deformación que afectó a las rocas mesozoicas de la región de la Mesa Central, fue la orogenia Laramide (Padilla y Sánchez, 1985; TristánGonzález et al., 1994; Centeno-García y Silva-Romo, 1997; Escalona-Alcázar et al., 2009). Las estructuras que se generaron durante este evento fueron foliación intensa y fallas inversas, resultado de un esfuerzo con orientación NE-SW (Tristán-González et al., 1994; Escalona-Alcázar et al., 2009). La finalización de este evento en la región de la Mesa Central es incierto, pero se puede inferir como Paleoceno temprano por la edad de la sedimentación continental de lechos rojos (Paleoceno tardío-Eoceno) que se encuentran en discordancia angular sobre los sedimentos mesozoicos deformados en varias localidades de la Mesa Central (Labarthe-Hernández et al., 1982; Nájera-Garza, 1997; Aranda-Gómez y McDowell, 1998). Otra guía para determinar este evento es la presencia de los intrusivos graníticos no deformados que se encuentran en la región de San Luis Potosí y Guanajuato con edades que varían entre 51 y 59 Ma (Mújica-Mondragón y Jacobo-Albarrán, 1983; Aranda-Gómez et al., 2007; Tristán-González, 2008).

En el norte de la ciudad de Zacatecas, las rocas de la secuencia de la UVS, están cruzadas por cuatro fallas principales: La Cantera, Mala Noche, Veta Grande y Pánuco, donde se alojaron la gran mayoría de vetas del DMZ. La orientación general de esas fallas es de $\mathrm{N} 50^{\circ}-60^{\circ} \mathrm{W}$ con inclinaciones de sus planos al SW y NE (Figuras 1 y 9).

La geometría de este arreglo de vetas refleja un desarrollo local de cizallamiento con zonas de dilatación en segmentos de las fallas, producto de un movimiento lateral derecho de las fallas principales. Este sentido se determinó por el arreglo en echelon de fracturas R que presenta el sistema de vetas principales y por estrías horizontales en los planos de falla.

De acuerdo a las observaciones de campo, relaciones estratigráficas y la edad más antigua de las rocas volcánicas del DMZ, se puede deducir que la formación de estas vetas debió ocurrir inmediatamente después de que cesaron los esfuerzos de compresión, anteriores al Eoceno temprano. En el campo se observó que este sistema de fallas fueron también los conductos para el emplazamiento de magmas félsicos. Las rocas más antiguas del DMZ son los volcaniclásticos Los Alamitos, cuya edad fue determinada por Ponce-Sibaja y Clark (1988) en 46.8 Ma por el método $\mathrm{K} /$ Ar en biotita, lo que los coloca en el Eoceno medio. De acuerdo con estos datos, la edad de estas fallas se puede considerar anterior al Eoceno medio.

\subsection{Falla La Cantera}

La falla La Cantera fue en algún tiempo la principal veta productora de plata en el DMZ; su longitud es de unos 11 $\mathrm{km}$, con anchura promedio de $20 \mathrm{~m}$. Tiene un rumbo general $\mathrm{N} 60^{\circ}-65^{\circ} \mathrm{W}$ con inclinación al $\mathrm{SW}$, sufriendo una inflexión a la altura del domo La Bufa (Figuras 2 y 9). En su primera fase de formación (post-laramídica y pre-Eoceno medio), su cinemática estuvo regida por movimiento derecho manifestado por las fracturas Riedel y estrías horizontales en 
su plano de falla, y es durante este evento que se emplazaron las primeras vetas de cuarzo. La reactivación de la falla ocurrió después del emplazamiento de la riolita La Bufa $(40.9 \pm 0.9 \mathrm{Ma})$, ya que el domo La Bufa se encuentra afectado por la falla La Cantera (etapa de extensión). En esta etapa su movimiento fue normal oblicuo izquierdo con un promedio de pitch de $58^{\circ} \mathrm{SE}$, e inclinación entre los $55^{\circ}$ y $80^{\circ} \mathrm{SW}$. Las estrías que se presentan sobre el cuarzo de la veta indican que fueron originadas en un evento de reactivación de la falla.

El bloque del piso de la falla La Cantera está formado por las rocas de la UVS y el bloque del techo en su segmento SE, por el conglomerado rojo de Zacatecas y rocas volcánicas del Eoceno (riolita Bufa). En su porción noroccidental corta a los sedimentos de la UVS así como a la Formación Zacatecas (zona de El Bote). El desplazamiento neto de la falla, estimado en obras mineras en su porción sureste, puede alcanzar $250 \mathrm{~m}$ (Stone, 1956). El plano principal de falla La Cantera se encuentra cortado y desplazado en algunos segmentos de su traza por fallas normales de alto ángulo y rumbo NNW que no continúan en el conglomerado Rojo de Zacatecas. A lo largo de la traza principal de la falla La Cantera se formaron escalones (zonas de dilatación), donde la veta se amplía formando cuerpos de cuarzo en forma sigmoide.

La lava que formó el domo La Bufa se emplazó donde la falla La Cantera muestra una inflexión hacia el SW, ocasionando el desarrollo de una zona de dilatación que facilitó la salida de la lava (Figuras 2 y 9). El domo La Bufa es una estructura elíptica, con eje mayor con rumbo E-W, paralelo al rumbo de la traza de la falla (Figuras 2 y 9). La falla La Cantera cortó al domo en su margen norte, donde formó una zona importante de argilización y silicificación (Figura 3A). Este domo tiene zonas muy silicificadas y fracturas verticales con óxidos de hierro que representan una actividad fumarólica importante (Figura 3B).

De acuerdo con las relaciones de campo, los domos y diques riolíticos del poniente de la sierra de Zacatecas se emplazaron siguiendo los patrones de fracturas $R$ de la falla
La Cantera (cerros El Magistral, La Valenciana, La Calavera, Figura 2). El cerro El Magistral es el dique mayor que aún conserva parte de la lava que formó el domo. De este dique se ramifican diques menores con orientación NNE, siguiendo fracturas $R^{\prime}$. Este dique coincide también con el contacto entre los sedimentos de la Formación Zacatecas y los de la UVS. La zona de vetas El Grillo, El Bote y El Orito siguieron patrones $R^{\prime}$. En la Figura 2, se muestra el esquema cinemático de las vetas y domos relacionados con la falla La Cantera.

\subsection{Falla Mala Noche.}

El sistema de vetas que forman la zona mineralizada de Mala Noche se localiza al norte de la veta La Cantera; tiene una longitud aproximada de unos $5 \mathrm{~km}$. Su segmento poniente tiene un rumbo $\mathrm{N} 75^{\circ} \mathrm{W}$, y en su porción oriente ENE, la inclinación de su plano de falla varía entre $60^{\circ} \mathrm{y}$ $80^{\circ} \mathrm{NNE}$. El cambio de rumbo de esta falla está marcado por una zona de dilatación de forma sigmoidal en la cual se desarrollaron múltiples vetas (Figuras 2 y 4). A lo largo de la veta Mala Noche se presenta un patrón múltiple de estructuras Riedel que indican un movimiento lateral derecho (Figura 4). En su extremo oriental, a la altura del tiro Bolado, la veta se ramifica formando un arreglo de "cola de caballo" con fallas normales de alto ángulo. En este segmento la falla Mala Noche tiene un rumbo $\mathrm{N} 70^{\circ} \mathrm{E}$ con inclinación de $70^{\circ} \mathrm{NW}$ y en su plano se observan estrías horizontales, surcos de acumulación de sílice, estructuras de media luna que señalan un movimiento lateral derecho (Figura 5A). Sobrepuestas a las estrías laterales, se aprecian estrías oblicuas que reflejan una reactivación por movimiento normal oblicuo-derecho, las cuales tienen un pitch de $70^{\circ} \mathrm{SE}$ (Figura 5B). Los domos y diques La Sierpe y San Gil de rumbo NNE se encuentran emplazados en las fracturas $R^{\prime}$ (Figura 2). El arreglo del juego de fracturas Riedel desarrolladas en la zona de cizalla de la falla, muestran que $\sigma_{1}$ tuvo una dirección NW-SE (Figura 4). Los domos y diques presentan silicificación intensa que
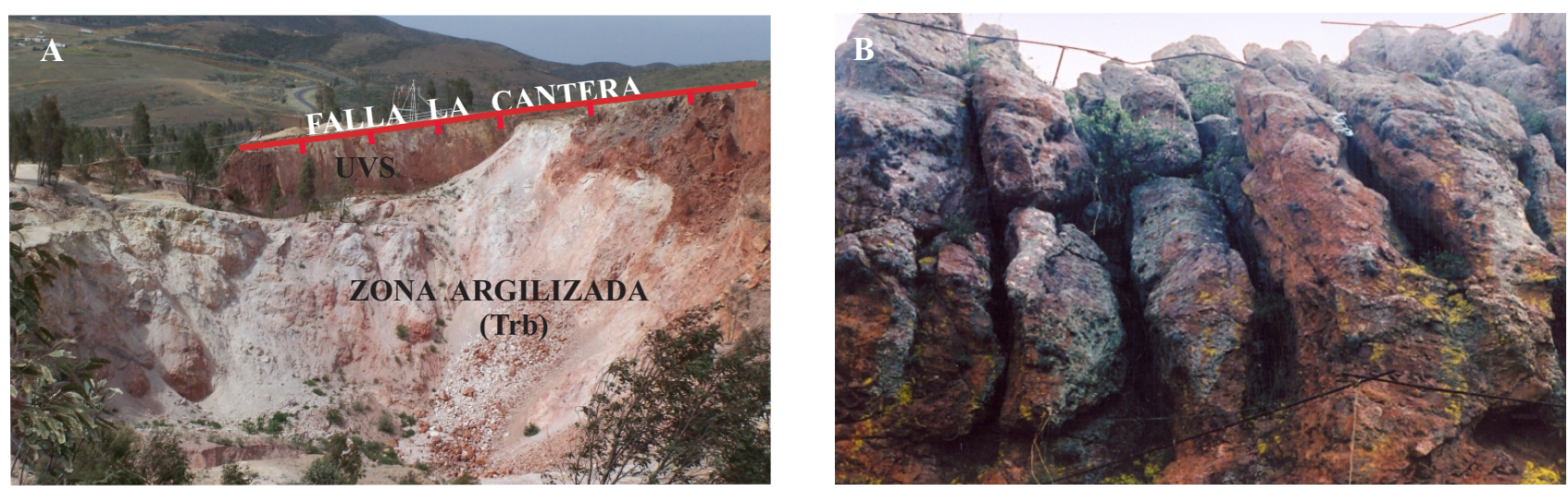

Figura 3. A). En la margen norte del domo La Bufa, en el alto de la Falla La Cantera, se desarrolló una zona de argilización y oxidación importante (vista al norte desde el mirador del Cerro La Bufa), B). En la porción central del domo del Cerro La Bufa se observan fracturas en el centro del domo, que sirvieron como canales de la actividad fumarólica con depositó óxidos de hierro. Trb-riolita Bufa, UVS-Unidad Vulcano-Sedimentaria 


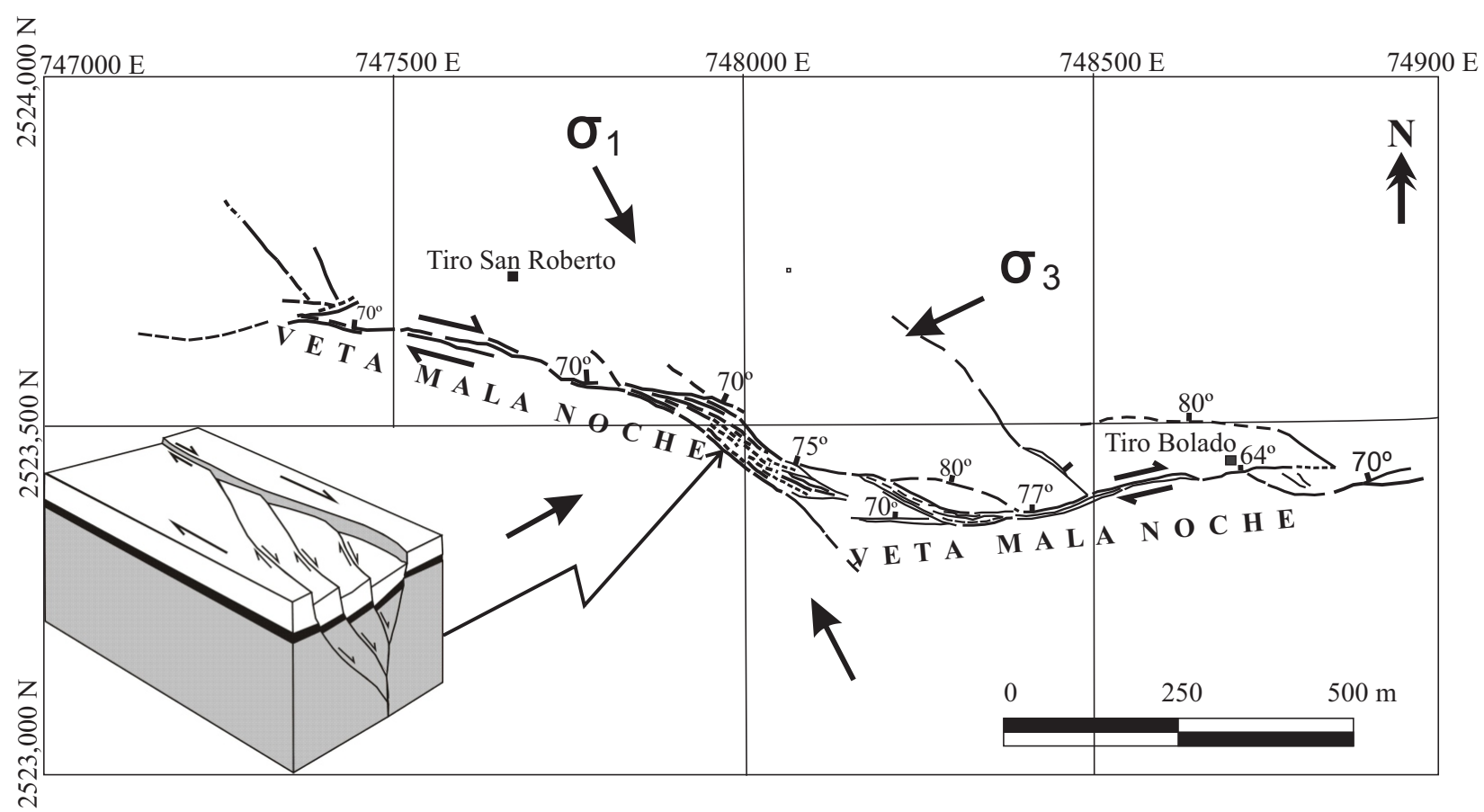

Figura 4. Mapa estructural de la Veta Mala Noche, en su parte media se aprecia la zona de dilatación principal en forma de sigmoide, donde se ha concentrado la actividad minera con mayor potencial minero. En el inserto de la parte inferior izquierda se muestra un modelo que explica la zona de dilatación producto de transtensión (con permiso de Capstone Gold, 2010).
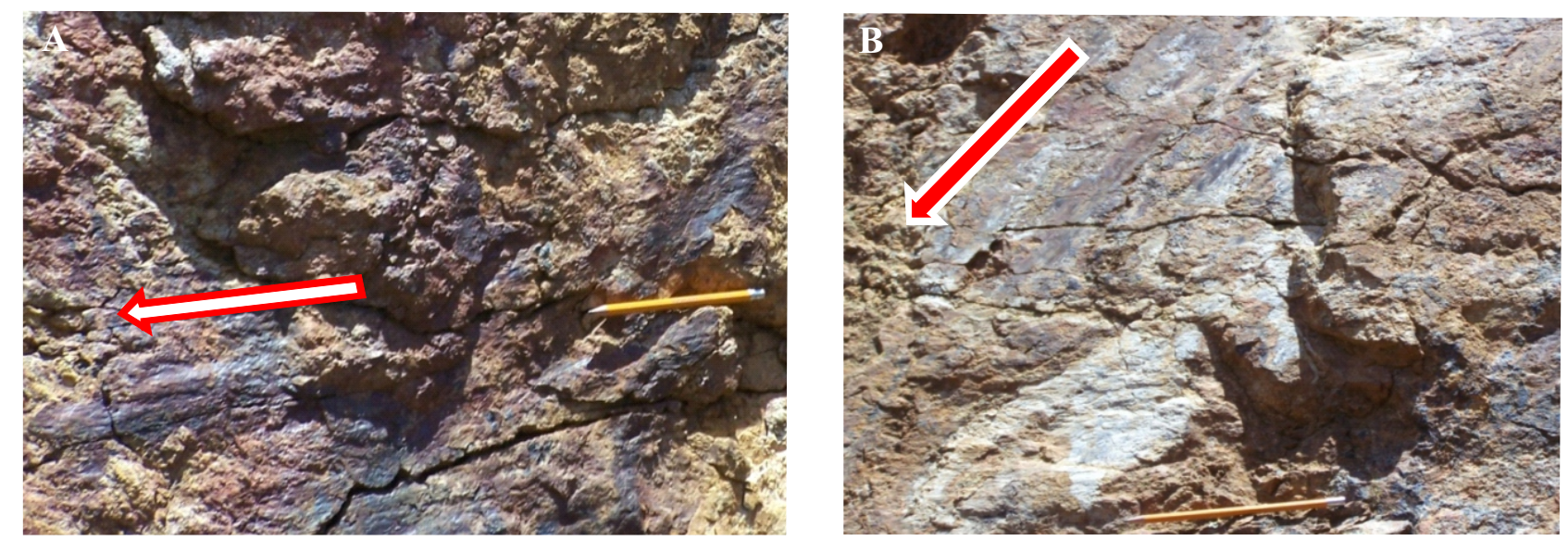

Figura 5. A). En la porción oriente de la Falla Mala Noche, se aprecia sobre el plano de falla la impresión de estrías horizontales, B) estrías oblicuas sobrepuestas a las horizontales.

se asocia a la última etapa de mineralización en el área, la cual se puede presentar en las márgenes de los diques $\mathrm{y}$ en las porciones centrales a profundidad, información proporcionada por la barrenación. El sistema de vetas de Mala Noche son rellenos de fisura de cuarzo amorfo con asociación de sulfuros, sobre todo de calcopirita, por lo que su producción principal es de cobre. La secuencia de rocas sedimentarias que encajona a estas vetas consiste principalmente de lutita, arenisca, andesita y algunos diques máficos pertenecientes a la UVS. La UVS se encuentra muy deformada, con desarrollo de zonas de cizalla producto de los múltiples despegues tectónicos intraformacionales y en cuyos planos de bajo ángulo se aloja mineralización.

\subsection{Falla Veta Grande.}

A unos $7 \mathrm{~km}$ al norte de la ciudad de Zacatecas se localiza la zona mineralizada Veta Grande (VG), donde actualmente solo se explota a escala pequeña. La zona de vetas es una franja de $1.5 \mathrm{~km}$ de ancho por unos $3 \mathrm{~km}$ de largo en las inmediaciones del poblado Veta Grande. En su porción sur-oriente, esta zona de vetas prácticamente 
desaparece y la mineralización se restringe solo a la traza de la VG. La mayoría de las vetas del área del poblado Veta Grande tienen un rumbo de $\mathrm{N} 45^{\circ}-60^{\circ} \mathrm{W}$ con echados al NE y SW. La VG tiene un rumbo promedio de $\mathrm{N} 50^{\circ}-55^{\circ} \mathrm{W}$ con inclinación promedio de $70^{\circ} \mathrm{SW}$ (Figuras 6 y 9). Su longitud es de aproximadamente $7 \mathrm{~km}$, con anchuras que varían de 1 a $10 \mathrm{~m}$. Al norte del poblado Veta Grande, la zona de cizalla de la falla tiene una anchura de unos $1500 \mathrm{~m}$, lo que corresponde a una zona de dilatación amplia, ya que hacia los extremos esta zona prácticamente desaparece (Figura 6).

El sistema secundario de vetas $R$, tiene un rumbo promedio de $\mathrm{N} 35^{\circ} \mathrm{W}$ con inclinaciones entre $60^{\circ}$ y $75^{\circ} \mathrm{SW}$, formando un ángulo promedio con $\mathrm{VG}$ de $20^{\circ}$. Las fracturas $R$ y $R^{\prime}$ fueron medidas en campo y están representadas en el estereograma inserto de la Figura 6, con planos de rumbo entre $\mathrm{N} 20^{\circ}-30^{\circ} \mathrm{E}$, en los que se observan estrías horizontales que reflejan un movimiento lateral derecho en su primera etapa de actividad (Figura 7A), y sobre de ellas están sobrepuestas estrías oblicuas las cuales tienen un pitch promedio de $65^{\circ}-70^{\circ} \mathrm{SE}$, lo que define a la falla como normal oblicua-derecha (Figura 7B). Las vetas están encajonadas en la UVS.

\subsection{Fallas Pánuco y Casa de Cerros}

El sistema de vetas asociado a las fallas Pánuco y Casa de Cerros se localiza en el extremo norte de la SZ (Figuras 1 y 9), y corresponde a vetas mineralizadas de menor longitud que las asociadas a los tres primeros sistemas de fallas ya mencionadas.

La veta Pánuco (VP) tiene un rumbo variable entre N35 $40^{\circ} \mathrm{W}$ con inclinación entre $60^{\circ}$ y $75^{\circ}$ al SW. Paralela a ella, hay fallas antitéticas con inclinación al NE (Figura 8A), con relleno de cuarzo de tonos azul pálido. Hay otro conjunto transversal de fallas-vetas que corresponden a fracturas $R^{\prime}$. La VP presenta en sus plano estrías horizontales que marcan un movimiento lateral derecho y las estrías oblicuas de segunda generación sobrepuestas sobre las horizontales, muestran que la VP en esta segunda etapa, se comportó como una falla normal-oblicua izquierda, con pitch de $75^{\circ}$ al SE. Actualmente las obras mineras en este sistema se encuentran inactivas.

El sistema de vetas Casa de Cerros es la última manifestación importante de vetas en el norte de la SZ. Es

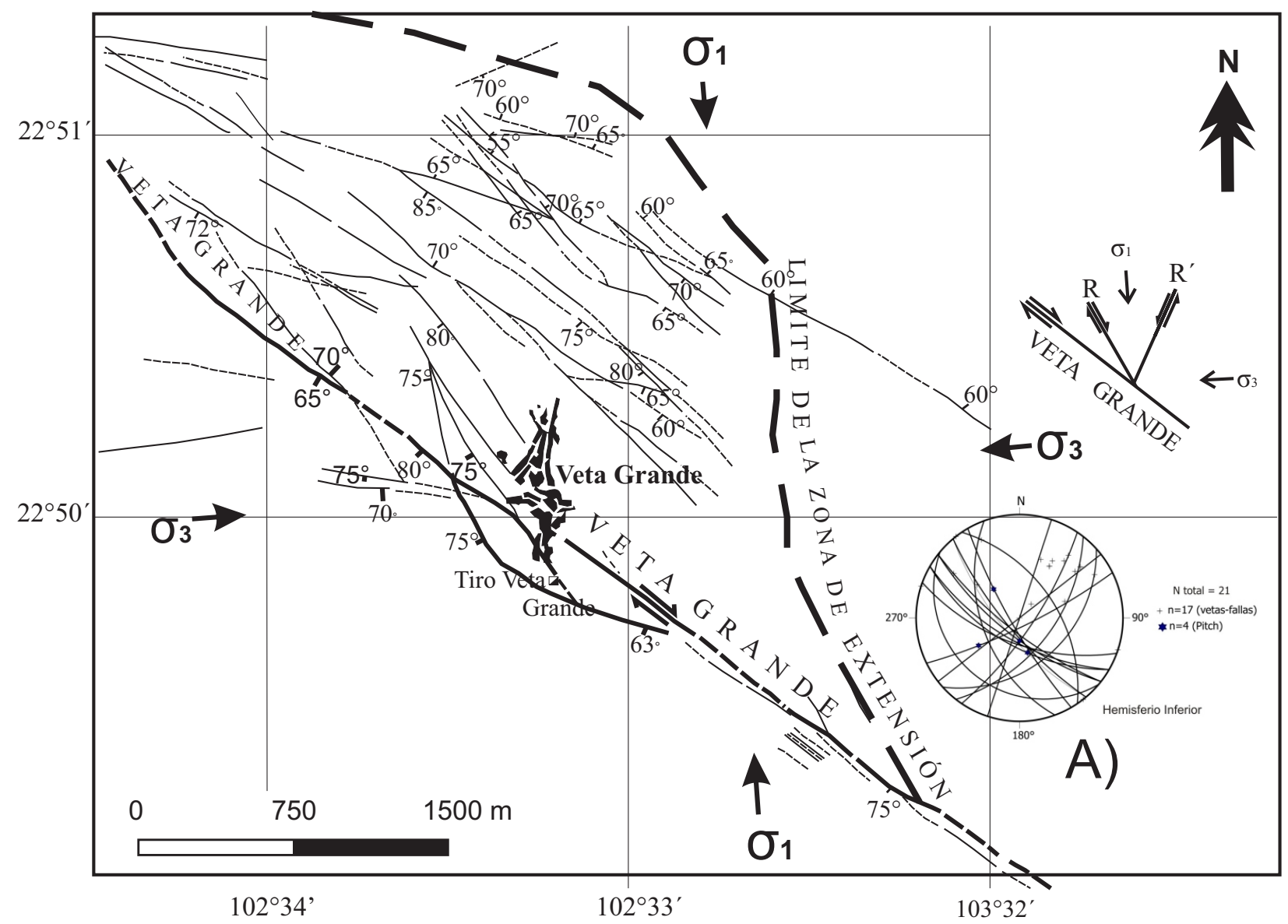

Figura 6, Mapa estructural de la zona de cizalla de Veta Grande, donde se observa una zona de dilatación importante al norte del poblado de Veta Grande, donde se ha concentrado la mayor parte de la actividad minera. Las fracturas $R$ están representadas en el estereograma inserto con datos de campo, con rumbo $\mathrm{N} 35^{\circ}-30^{\circ} \mathrm{W}$ y las $R^{\prime}$ con rumbo entre $\mathrm{N} 20^{\circ}-30^{\circ} \mathrm{E}$. Los planos representados en el estereograma con inclinación al SW corresponden a segmentos medidos sobre la traza de VG y a fracturas R (modificado de Pérez-Martínez et al., 1961). 

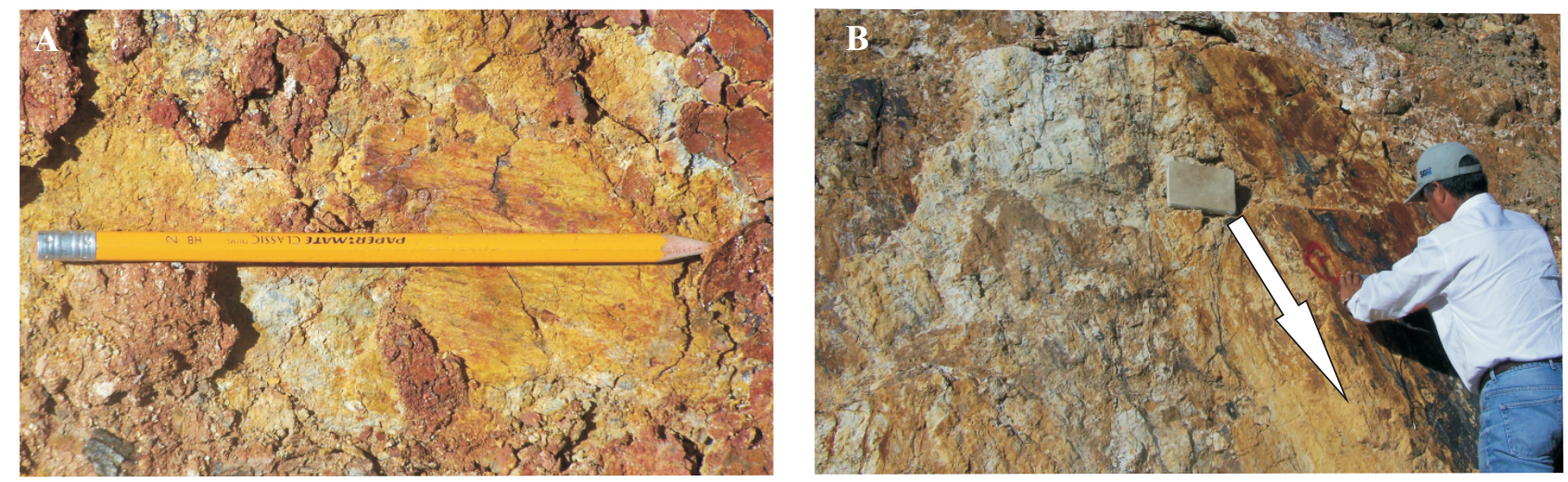

Figura 7. A). Fotografía tomada sobre un segmento de la falla Veta Grande donde se aprecian las estrías desarrolladas en su primera fase de formación en posición horizontal, B). En el mismo plano de falla, se sobrepusieron las estrías oblicuas con pitch de $65^{\circ} \mathrm{SE}$, como consecuencia de la reactivación de la falla en su segunda etapa.
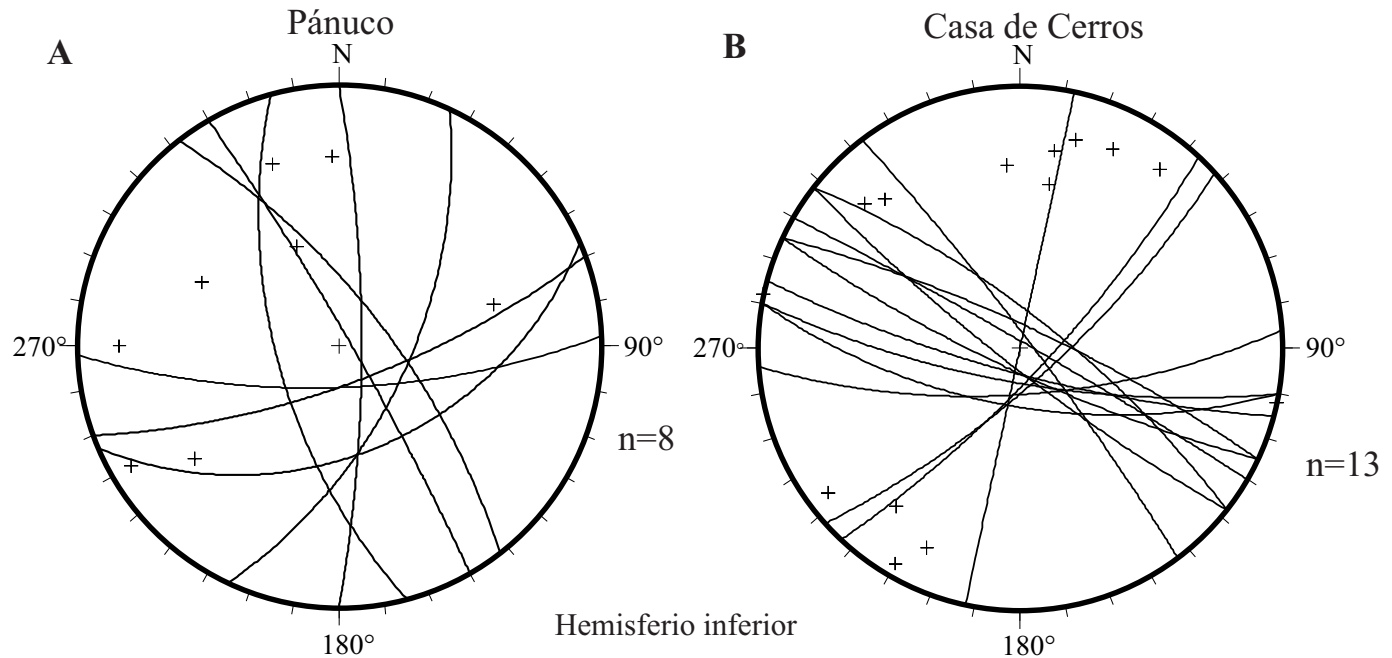

Stereo32, Unregistered Version

Figura 8. A) Estereograma que muestra los planos de vetas de la zona de Panuco, B) estereograma de planos de vetas del sistema de Casa de Cerros. Las cuales muestran el arreglo de fracturas Riedel parecido a las de las vetas La Cantera, Mala Noche y Veta Grande.

un conjunto de fallas-veta de alto ángulo, entre $70^{\circ}$ y $85^{\circ}$ cuyos planos buzan tanto al $\mathrm{SW}$ como al NE, con un rumbo que oscila entre $\mathrm{N} 50^{\circ}-80^{\circ} \mathrm{W}$, con estrías horizontales que muestran movimiento derecho e izquierdo. También se presenta un juego transversal de fallas de rumbo $\mathrm{N} 45^{\circ} \mathrm{E}$ de alto ángulo, de $75^{\circ}$ a $80^{\circ} \mathrm{SE}$ (Figura 8B). En la actualidad las obras mineras que se han desarrollado sobre este sistema se han hecho con fines de exploración, a excepción de su extremo sureste donde hay actividad minera.

\section{Discusión y Conclusiones}

En trabajos previos se sugirió un modelo de caldera para explicar el origen de las rocas volcánicas y yacimientos minerales en el DMZ, donde se consideró a los domos e ignimbritas que afloran en los alrededores de la ciudad de Zacatecas, como rocas asociadas a una caldera de colapso (Ponce-Sibaja y Clark, 1988). Sin embargo, la distribución no circular de los domos no es coherente con un modelo de caldera, y a la fecha no se ha documentado que la fuente de las ignimbritas localizadas al norte de la ciudad de Zacatecas, pertenezcan a esta caldera, sino que más bien pertenecen a flujos distantes que arribaron a la zona procedentes de fuentes de la SMO (Tristán-González, 2008). Por otro lado, se documenta en este trabajo que algunas de las estructuras (fallas y fracturas) controlaron el emplazamiento de domos y diques félsicos (p.ej. domos y diques en el sistema de fallas La Cantera). Resulta difícil acomodar las fallas La Cantera, Mala Noche, Veta Grande y Pánuco-Casa de Cerros de orientación NW-SE (Figura 9), en un modelo semicircular de fracturas radiales o concéntricas asignable al desarrollo de una caldera de colapso. Ni los espesores ni la distribución de las ignimbritas se ajustan a 


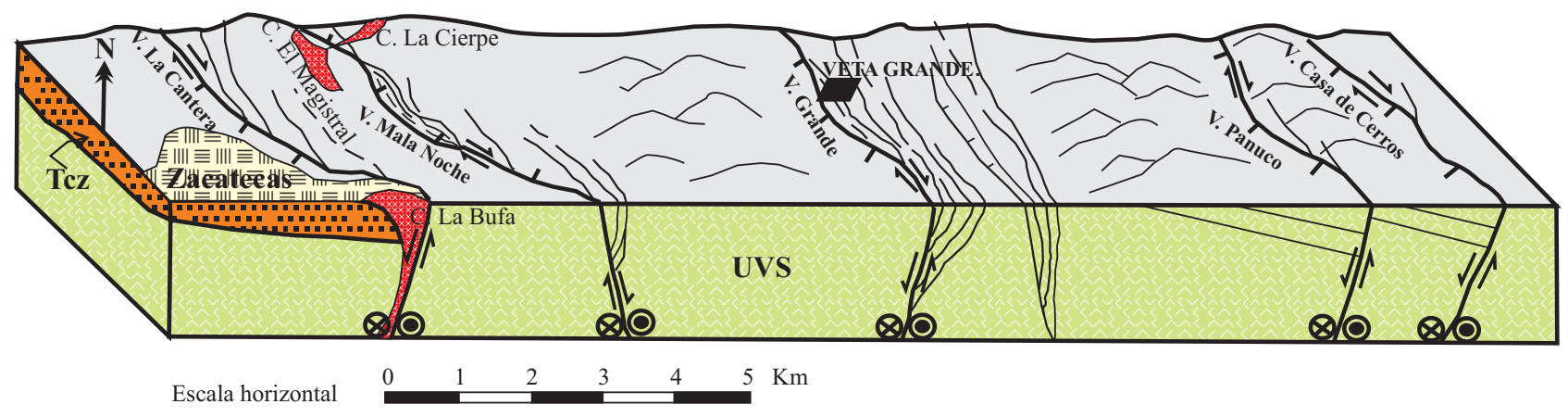

Figura 9. Bloque diagramático donde se representan los cuatro sistemas de vetas del distrito Minero de Zacatecas. En tamizado rojo se muestran los domos félsicos de la riolita Bufa. Tcz- conglomerado rojo de Zacatecas, UVS-Unidad Vulcano Sedimentaria.

este modelo, aun considerando el profundo nivel de erosión que plantean Ponce-Sibaja y Clark (1988). Este hecho expondría en superficie a diques alimentadores piroclásticos de las ignimbritas (lo cual no se ha documentado hasta ahora en esta zona de Zacatecas), y estos tenderían a delinear los límites de la zona de colapso. En contraparte, las fallas $\mathrm{y}$ fracturas del DMZ tienen un arreglo que es coherente con un sistema regional de fallamiento NW-SE (Figura 9), que ha sido denominado San Luis-Tepehuanes (NietoSamaniego et al., 2005; Loza-Aguirre, 2005; Loza-Aguirre et al., 2008), aunque dichos autores no mencionan que las fallas en este segmento del sistema presenten evidencias de movimiento lateral.

Evidencias de un primer evento de fallamiento formado por actividad lateral de las fallas principales de rumbo NWSE invariablemente se han considerado como netamente resultado de esfuerzos extensionales con dirección NESW ocurridas dentro del Cenozoico (Loza-Aguirre et al., 2008). En los trabajos sobre la presencia del sistema de Fallas San Luis-Tepehuanes no se menciona la actividad de movimiento lateral (Nieto-Samaniego et al., 2005; LozaAguirre, 2005; Loza-Aguirre et al., 2008). La presencia de dos juegos de estrías en los planos de falla acompañados de otros indicadores cinemáticos asociados, señalan dos eventos de fallamiento: el primero de carácter lateral derecho y el segundo de carácter normal desarrollado por esfuerzos de extensión que retomaron los planos de fallas generados durante la primera etapa de fallamiento.

En algunos trabajos de interpretación de imágenes de satélite realizados para compañías mineras por empresas privadas (p. ej. los trabajos de ERA-MAPTEC) que permanecen como reportes internos en unidades mineras, señalan que el arreglo geométrico de las fallas en el DMZ sugieren un control de mineralización por zonas de dilatación derivadas de movimientos laterales. Sin embargo, los datos estructurales medidos en los planos de falla y la presencia de grabens y medios grabens delimitados por esas fallas, prevalecieron en los diferentes trabajos de cartografía para asignar la presencia de estructuras generadas por esfuerzos puramente extensionales. Por otro lado, el reconocimiento de la existencia de los dos registros de actividad en los planos de falla pueden explicar la geometría (inflexiones, escalonamientos e interrupciones) que se observan a lo largo de las trazas de las fallas principales, las cuales son características de fallamiento lateral, atestiguadas por la presencia de estrías horizontales, surcos en forma de "V" generados en los planos de falla, cristales de cuarzo, escalones y estructuras de media luna marcadas en la acumulación de minerales en los planos de falla que indican un movimiento horizontal. Otro argumento de que este evento de fallamiento lateral fue primero, es que en los puntos de inflexión de algunas de estas estructuras, donde se generaron esfuerzos de transtensión, se formaron estructuras de acomodo escalonadas de bloques en cuyos límites se emplazaron vetas mineralizadas y el emplazamiento de magma félsico para originar domos y diques (p. ej. domo La Bufa en la falla La Cantera, Figura 9). Las estructuras secundarias relacionadas a las fallas principales, por su posición, características y geometría, son coherentes con sistemas de fracturas Riedel $R$ y $R^{\prime}$, desarrolladas mecánicamente durante el movimiento de cizalla horizontal entre los bloques, los que son muy claros en la zona de la veta Mala Noche (Figura 9).

La temporalidad relativa del segundo evento de fallamiento y el carácter del mismo, está dado porque las estrías (pitch $60^{\circ}-70^{\circ} \mathrm{SE}$ ) generadas en los planos de falla sobre el material de la veta de cuarzo. Estas estrías son más visibles sobre el material de la veta La Cantera, mientras que las laterales se aprecian mejor en los respaldos de la roca. Este mismo orden temporal de los eventos de las fallas está sugerido por el fallamiento normal que corta a algunos de los domos emplazados durante la primera etapa de fallamiento (p. ej. la falla La Cantera cortó al domo La Bufa en su parte norte, Figura 9). El mecanismo de emplazamiento de domos en ambientes transtensivos ha sido discutido por Alaniz-Álvarez et al. (2002), argumentado por Tristán-González (2008) y Aguillón-Robles et al. (2009) para la porción sur-central de la Mesa Central entre San Luis Potosí, Guanajuato y Zacatecas. Un tercer evento de fallamiento extensional posterior que aquí no se 
discute, generó las estructuras N-S que delimitan el horst que constituye la parte norte de la SZ. Como conclusión, se argumenta en este trabajo que el sistema de fallas del DMZ registra en sus planos las evidencias de dos sistemas de fallamiento: el primero de carácter transpresivo y el segundo, extensional, documentado por la edad de las rocas volcánicas más antiguas del DMZ y la edad de la extensión del Oligoceno de la MC.

El emplazamiento de diques riolíticos y domos asociados estuvo ligado a la primera etapa de fallamiento y mineralización y ambos factores (magmatismo y fallamiento) generaron la primera etapa de mineralización.

La segunda etapa de fallamiento retomó las estructuras principales definidas en la primera, y por lo tanto una segunda etapa de mineralización determinada por barrenación en la zona de rocas volcánicas de la ignimbrita La Virgen en el sur del DMZ.

El arreglo de vetas mineralizadas en el DMZ es más coherente considerando esta primera etapa de fallamiento como resultado de una actividad lateral ocurrida como una etapa de transición entre la orogenia Laramide y el fallamiento tipo Cuencas y Sierras. Tomando en cuenta estos criterios se puede guiar mejor las futuras exploraciones en el DMZ.

\section{Agradecimientos}

Los autores agradecen al Ing. Mariano Carrizales Ibarra, Superintendente de Geología de la Cía. Minera Capstone Gold S.A. de C.V. por sus facilidades para conocer la geología de la zona de la mina Mala Noche. Al Dr. Rafael Barboza Gudiño por su apoyo y comentarios para realizar este trabajo dentro de las investigaciones internas del Instituto de Geología de la Universidad Autónoma de San Luis Potosí. A la Dra. Fernanda Campa y al Dr. Roberto S. Molina Garza, por la revisión del manuscrito y sus valiosas aportaciones y comentarios. Al Ing. Erasmo Mata Martínez por su apoyo en la preparación de muestras para fechamiento. Este estudio fue financiado en parte con fondos del proyecto CONACyT CB-83700 a cargo del Dr. J. Ramón Torres Hernández.

\section{Referencias}

Aguillón-Robles, A., Tristán-González, M., Aguirre-Díaz, G.J., Bellon, H., 2009, Syn-extensional intra-plate trachydacite-rhyolitic dome volcanism of the Mesa Central, southern Sierra Madre Occidental Volcanic Province, Mexico: Journal of Volcanology and Geothermal Research, 187, 33-52.

Alaniz-Álvarez, S.A., Nieto-Samaniego, A.F., Morán-Centeno, D.J., AlbaAldave, L., 2002, Rhyolitic volcanism in extension zone associated with strike-slip tectonics in the Taxco region, southern Mexico: Journal of Volcanology and Geothermal Research, 118, 1-14.

Aranda-Gómez, J.J., McDowell, F.W., 1998, Paleogene in the southerm Basin and Range Province of Mexico: Syndepositional tilting of
Eocene red beds and Oligocene volcanic rocks in the Guanajuato Mining District: International Geology Review, 40, 116-134.

Aranda-Gómez, J.J., Henry, C.D., Luhr, J.F., 2000, Evolución tectonomagmática post-paleocénica de la Sierra Madre Occidental y de la porción meridional de la provincia tectónica de Cuencas y Sierras, México: Boletín de la Sociedad Geológica Mexicana, 53(1), 59-71.

Aranda-Gómez, J.J., Molina-Garza, R., McDowell, F.W., Vassallo-Morales, L.F., Ortega-Rivera, M.A., Solorio-Munguía, J.G., Aguillón-Robles, A., 2007, The relationships between volcanism and extension in the Mesa Central: the case of Pinos, Zacatecas, Mexico: Revista Mexicana de Ciencias Geológicas, 24, 216-233.

Bastin, E.S., 1941, Paragenetic relations in the silver ores of Zacatecas, Mexico: Economic Geology, 36, 371-400.

Botsford, C.W., 1909, The Zacatecas district and its relations to Guanajuato and other camps: Engineering and Mineral Journal, 87, 80-95.

Burckhardt, C., 1906, Sobre el descubrimiento del trías marino en Zacatecas: Boletín de la Sociedad Geológica Mexicana, 2, 43-45.

Burckhardt, C., Scalia, S., 1906, Geologie des environs de Zacatecas: Guide des excursions: Décimo Congreso Geológico Internacional, México, Distrito Federal, 16, 1-26.

Caballero-Martínez, J.A., Blanco; J.I., Luevano-Pinedo, A., 1999, Carta Geológico Minera, Zacatecas, F13B58, 1:50000: Servicio Geológico Mexicano (SGM), 1 mapa.

Campa-Uranga, M.F., Coney, P.J., 1983, Tectono-stratigraphic terranes and mineral resource distribution in Mexico: Canadian Journal of Earth Sciences, 20, 1040-1051.

Centeno-García, E., Silva-Romo, G., 1997, Petrogenesis and tectonic evolution of central Mexico during Triassic-Jurassic time: Revista Mexicana de Ciencias Geológicas, 14, 244-260.

Cox, A., Dalrymple, G.B., 1967, Statistical analysis of geomagnetic reversal data and the precision of potassium-argon dating: Journal of Geophysical Research, 72, 2603-2614.

de Cserna, Z., 1976, Geology of the Fresnillo area, Zacatecas, México: Geological Society of America Bulletin, 87(8), 1191-1199.

Edwards, J. D., 1955, Studies of some early Tertiary red conglomerates of Central Mexico: United States Geological Survey Professional Paper, 264-H, 153-185.

Escalona-Alcázar, F.J., Delgado-Argote, L.A., Weber, B., Núñez-Peña, E.P., Valencia, A.B., Ortiz-Acevedo, O., 2009, Kinematics and U-Pb dating of detrital zircons from the Sierra de Zacatecas, Mexico: Revista Mexicana de Ciencias Geológicas, 26(1), 48-64.

Gutiérrez-Amador, M., 1908, Las capas cárnicas de Zacatecas: Boletín de la Sociedad Geológica Mexicana, 4, 29-35.

Henry, C.D., Aranda-Gómez, J.J., 1992, The real southern Basin and Range: Mid to late Cenozoic extension in Mexico: Geology, 20, 701-704.

Labarthe-Hernández, G., Tristán-González, M., Aranda-Gómez, J.J., 1982, Revisión estratigráfica del Cenozoico de la parte central del Estado de San Luis Potosí: Universidad Autónoma de San Luis Potosí, Instituto de Geología y Metalurgia, Folleto técnico, 85, 208 p.

Loza-Aguirre, I., 2005, Estudio estructural de la actividad cenozoica del sistema de fallas San Luis Potosí-Tepehuanes de la región ZacatecasSan José de Gracia, México: Ciudad Madero, Tamaulipas, México, Instituto Tecnológico de Ciudad Madero, tesis profesional, $95 \mathrm{p}$.

Loza-Aguirre, I., Nieto-Samaniego, A.F., Alaniz-Álvarez, S.A., Iriondo, A., 2008, Relaciones estratigráfico-estructurales en la intersección del sistema de fallas San Luis-Tepehuanes y el graben de Aguascalientes, México central: Revista Mexicana de Ciencias Geológicas, 25(3), 533-548.

Maldonado-Koerdell, M., 1948, Nuevos datos geológicos y paleontológicos sobre el Triásico de Zacatecas: Anales de la Escuela Nacional de Ciencias Biológicas, 5(3-4), 291-294.

Mapes, V.E., 1949, Los Criaderos Minerales de "El Bote", Zacatecas, México: Instituto Nacional de Investigación de Recursos Minerales, México, 24, 1-39.

McGehee, R.V., 1976, Las rocas metamórficas del arroyo de La Pimienta Zacatecas, Zacatecas: Boletín de la Sociedad Geológica Mexicana, 37(1), 1-10. 
Monod, O., Calvert, P.H., 1992, Structural and stratigraphy reinterpretation of the Triassic units near Zacatecas, Zacatecas, central Mexico: Evidence of Laramide nappe pile: Zentralblatt für Geologie und Paläontologie, 6, 1533-1544.

Mújica-Mondragón, R., Jacobo-Albarrán, J., 1983, Estudio petrogenético de las rocas ígneas y metamórficas del Altiplano Mexicano, México: Instituto Mexicano del Petróleo, Proyecto C-1156, 78 p.

Nájera-Garza, J., 1997, Study on the origin and geologic and metallogenetic significance of the Zacatecas red conglomerate, Zacatecas, Mexico, en II Convención sobre la Evolución Geológica de México, Pachuca, Hidalgo, México, 47.

Nieto-Samaniego, A.F., Ferrari, L., Alaniz-Álvarez, S.A., LabartheHernández, G., Rosas-Elguera, J.G., 1999, Variation of Cenozoic extension and volcanism across the southern Sierra Madre Occidental Volcanic Province, Mexico: Geological Society of American Bulletin, 111(3), 347-363.

Nieto-Samaniego A.F, Alaniz-Álvarez, S.A., Camprubí, A., 2005, La Mesa Central de México: estratigrafía, estructura y evolución tectónica cenozoica: Boletín de la Sociedad Geológica Mexicana, 52(3), 285-318.

Ocejo-Pérez, T., 1979, Estudio geológico superficial del distrito minero de Zacatecas: San Luis Potosí, México, Universidad Autónoma de San Luis Potosí, tesis profesional, 123 p.

Padilla y Sánchez, R.J., 1985, Las estructuras de la curvatura de Monterrey, estados de Coahuila, Nuevo León, Zacatecas y San Luis Potosí: Universidad Nacional Autónoma de México, Instituto de Geología, Boletín, 6, 1-20.

Pérez-Martínez, J., Mapez-Vásquez, E., Pesquera-Velázquez, R., 1961, Bosquejo geológico del distrito minero de Zacatecas: Consejo de Recursos Naturales No Renovables, Boletín, 52, 1-38.

Ponce-Sibaja, 1985, Caldera development and economic mineralization in the Zacatecas mining district, Zacatecas, Mexico: El Paso, University of Texas, M.S. Thesis, $267 \mathrm{p}$.

Ponce-Sibaja, B., Clark, K.F., 1988, The Zacatecas Mining District: A Tertiary caldera complex associated with precious and base metal mineralization: Economic Geology, 83, 1668-1682.

Ranson, W.A., 1975, Geology and Petrology of portions of the Zacatecas and Guadalupe quadrangles, Zacatecas, Mexico: University New Orleans, M.S. Thesis, 83 p.

Stewart, J.H., 1978, Basin and Range structure in western North America, a review: Geological Society of America Memoir, 152, 1-30.
Stewart, J.H., 1998, Regional characteristics, tilt domains, and extensional history of the late Cenozoic Basin and Range province, western North America: Geological Society of America Special Paper, $323,47-74$.

Stone, J.G., 1956, Geology and the ore deposits of the Cantera Mine, Zacatecas, Mexico: Economic Geology, 51(1), 80-95.

Torres-Hernández, J. R., Tristán-González, M., Labarthe-Hernández, G., López-Doncel., Aranda-Gómez, J.J., McDowell, F., Ortega, A., Vasallo, L., Solorio; J. G., 2000, Conglomerados Rojos del Centro de México y vulcanismo asociado: Indicadores de extensión episódica durante el Terciario temprano y medio (resúmenes), en II Reunión Nacional de Ciencias de la Tierra, Unión Geofísica Mexicana, Puerto Vallarta, Jalisco, 20(3), 157-158.

Tristán-González, M., 2008, Evolución tectono-volcánica durante el Paleógeno en la porción sur-oriental de la Mesa Central: Juriquilla, Querétaro, México: Universidad Nacional Autónoma de México, Centro de Geociencias, tesis doctoral, $207 \mathrm{p}$.

Tristán-González, M., Torres-Hernández, J.R., 2000, Carta Geológica Minera y Geoquímica de San Luis Potosí y Zacatecas: Secretaría de Comercio y Fomento Industrial, Consejo de Recursos Minerales, 65 p., 1 mapa.

Tristán-González, M., Torres-Hernández, J.R., Mata-Segura, J.L., 1994, Correlación estratigráfica de la geología de la Mina Proaño y Plateros, Zacatecas, con otras localidades del Terreno Guerrero al suroriente del Estado de Zacatecas: Universidad Autónoma de San Luis Potosí, Instituto de Geología, no publicada, $97 \mathrm{p}$.

Tristán-González, M., Aguillón-Robles, A., Barboza-Gudiño, J.R., TorresHernández, J.R., Bellon, H., López-Doncel, R.A., Rodríguez-Ríos, R., Labarthe-Hernández, G., 2009, Geocronología y distribución espacial del Campo Volcánico de San Luis Potosí: Boletín de la Sociedad Geológica Mexica, 61 (3), 287-303.

Yta, M., 1992, Étude geodynamique et metallogenique d'un secteur de la "Faja de Plata", Mexique. La zone de Zacatecas-Francisco I Madero-Saucito: L'Universite D' Orleans, Docteur de L'Universitte D'Orleans, These, $270 \mathrm{p}$.

Manuscrito recibido: Noviembre 3, 2010.

Manuscrito corregido recibido: Febrero 5, 2011.

Manuscrito aceptado: Marzo 20, 2011. 